\title{
Micromechanical Formulation of the Yield Surface in the Plasticity of Granular Materials
}

\author{
Homayoun Shaverdi, ${ }^{1}$ Mohd. Raihan Taha, ${ }^{1}$ and Farzin Kalantary ${ }^{2}$ \\ ${ }^{1}$ Universiti Kebangsaan Malaysia (UKM), 43600 Bandar Baru Bangi, Selangor, Malaysia \\ ${ }^{2}$ Department of Geotechnical Engineering, Faculty of Civil Engineering, K. N. Toosi University of Technology, \\ 1346 Vali Asr Street, Vanak, Tehran 19697, Iran
}

Correspondence should be addressed to Homayoun Shaverdi; homayoun_shaverdi@yahoo.com

Received 7 June 2013; Revised 31 July 2013; Accepted 15 August 2013

Academic Editor: Pengcheng Fu

Copyright (C) 2013 Homayoun Shaverdi et al. This is an open access article distributed under the Creative Commons Attribution License, which permits unrestricted use, distribution, and reproduction in any medium, provided the original work is properly cited.

An equation is proposed to unify the yield surface of granular materials by incorporating the fabric and its evolution. In microlevel analysis by employing a Fourier series that was developed to model fabric, it is directly included in the strength of granular materials. Inherent anisotropy is defined as a noncoaxiality between deposition angle and principal compressive stress. Stressinduced anisotropy is defined by the degree of anisotropy $\alpha$ and the major direction of the contact normals. The difference between samples which have the same density (or void ratio) but different bedding angles is attributed to this equation. The validity of the formulation is verified by comparison with experimental data.

\section{Introduction}

There are numerous experimental observations showing that the shape of the failure surface for soils is influenced by the microstructural arrangement (or fabric) (e.g., [1-3]). It has long been known that the failure condition is influenced by the microstructural arrangement of the constituent particles. Several expressions for failure criteria have been proposed to include the effect of fabric and its evolution. Baker and Desai [4] proposed the so-called joint isotropic invariants of stress and appropriate anisotropic tensorial entities. Pastor [5], by using this method, proposed a constitutive model to account for fabric anisotropy.

Pietruszczak and Mroz [6] related inherent anisotropy to the microstructural arrangement within the representative volume of material. They used a second-order tensor whose eigenvectors specify the orientation of the axes of the material symmetry. The failure criteria proposed by Pietruszczak and Mroz [6] were formulated in terms of the stress state and a microstructure tensor. Lade [3], by using the method proposed by Pietruszczak and Mroz [6], related the loading directions to the principal directions of the cross-anisotropic microstructure arrangement of the particles.
In order to connect the microscopic character of the granular materials with overall macroscopic anisotropy, various quantities have been proposed; for example, Oda [1], Oda et al. [2], and Oda [7] defined the fabric of anisotropy by using the distribution of the unit contact normals. Mehrabadi et al. [8] defined another microstructural arrangement and connected these parameters to the overall stress and other mechanical characteristics of granular materials. Gao et al. [9] and Gao and Zhao [10] proposed a generalized anisotropic failure criterion through developing an isotropic failure criterion by introducing two variables to account for fabric anisotropy. The first one is the fabric anisotropy that was proposed by Oda and Nakayama [11] and the second one is the joint invariants of the deviatoric stress tensor and the deviatoric fabric tensor to characterize the relative orientation between stress direction and fabric anisotropy. They related the frictional coefficient $\eta^{p}$ to the anisotropic variable $A$. Fu and Dafalias [12] showed that there is a difference between friction angle in the isotropic and anisotropic cases. In the isotropic case, friction angle would be a directionindependent constant, while in the anisotropic case, it is a function of the bedding angle with respect to the shear plane (in the Mohr-Coulomb failure criterion). Fu and Dafalias [13] 
by using discrete element method (DEM) investigated the effect of fabric on the shear strength of granular materials. They proposed an anisotropic shear failure criterion on the basis of noncoaxiality between the bedding plane orientation and the shear plane. The inherent fabric anisotropy was taken into account by considering the orientation of the bedding plane with respect to the principal stress axes.

The specification of the condition at failure for anisotropic granular soils constitutes an important problem and numerous criteria have been proposed in the past. In this paper, we endeavor to incorporate the effect of inherent and induced anisotropy in the yield surface. The inherent and induced anisotropies are expressed as explicit functions of the bedding angle $\beta$ and the magnitude of anisotropy $\alpha$ (in the distribution of contact normals). These two elements (inherent and induced anisotropy) are combined, and the Mohr-Coulomb yield surface which is modified to account for the kinematic yield surface [14-16] is developed by including the fabric and its evolution. The equation of the yield surface that is proposed for granular soils is compared with the experimental results from Oda et al. [17]. It shows that the equation is able to capture the shearing behavior of soils with different bedding angles.

\section{Definition of Inherent Anisotropy}

Inherent anisotropy is attributed to the deposition and orientation of the long axes of particles $[1,2,7]$. Oda et al. [17] and Yoshimine et al. [18] showed that the drained and undrained response of sand and approaching the critical state failure are actually affected by the direction of the principal stress relative to the orientation of the soil sample. Pietruszczak and Mroz [6] included the effect of fabric by the following equation:

$$
F=\tau-\eta g(\theta) p_{o}
$$

where $\tau=J_{2}^{1 / 2}$ is the second invariant of the stress tensor, $p_{o}=\operatorname{tr} \sigma / 3$ is first invariant of the stress tensor, $g(\theta)$ is Lode's angle, and $\eta$ is a constant for isotropic materials and defined by the following equation for anisotropic materials:

$$
\eta=\eta_{o}\left(1+\Omega_{i j} l_{i} l_{j}\right)
$$

where $\eta_{o}$ is the constant material parameter, $\Omega_{i j}$ describes the bias in material microstructure spatial distribution, and $l_{i}$ and $l_{j}$ are the loading directions. Lade [3] by using these formulations proposed a failure criterion for anisotropic materials. Wan and Guo [19] accounted for the effect of inherent anisotropy in microlevel analysis by the ratio of projection of major-to-minor principal values of the fabric tensor along the direction of the principal stresses. Li and Dafalias $[20,21]$ incorporated this effect by the fabric tensor which was proposed by Oda and Nakayama [11]. These two methods used the same basic approach; they used the principal values of the fabric tensor in their formulations. However, micromechanical studies $[2,11]$ have shown that in the shearing process, the preferred orientation of the particles in a granular mass may undergo only small changes. Its value may well endure after the onset of the critical state; hence, the fabric anisotropy renders the locus of the critical state line. In this paper, $\cos 2\left(\beta_{i}-\beta_{\mathrm{o}}\right)$ is used to model the effect of inherent anisotropy. $\beta_{i}$ indicates the variation of the long axes of particles with respect to the major principal stress; $\beta$ 。 is the angle of deposition with respect to the major principal stress. Hence,

$$
\left(\frac{\sigma_{1}}{\sigma_{2}}\right)_{f} \propto \cos 2\left(\beta_{i}-\beta_{\circ}\right)
$$

\section{Definition of Stress-Induced Anisotropy}

With increasing shear loads, the contact normals tend to concentrate in the direction of the major compressive stress. Contacts are generated in the compressive direction and disrupted in the tensile direction. These disruption and generation of the contact normals are the main causes of the induced anisotropy in the granular materials [2]. In order to include the fabric evolution (or induced anisotropy), a function in which changes of the contact normals are included must be defined. Wan and Guo [19] used the following equation:

$$
\dot{F}_{i j}=x \dot{\eta}_{i j}
$$

where $\dot{F}_{i j}$ shows the evolution of fabric anisotropy, $x$ is a constant, and $\dot{\eta}_{i j}$ is the ratio of the shear stress to the confining pressure, or $\eta=(q / p)$. Dafalias and Manzari [22] related the evolution of fabric to the volumetric strain in the dilatancy equation. The evolution of fabric comes to play only after dilation. Based on DEM simulation presented by Fu and Dafalias [12], Li and Dafalias [23] developed an earlier model (yield surface) to account for fabric and its evolution in a new manner by considering the evolution of fabric tensor towards its critical value.

By using Fourier series, Rothenburg and Bathurst [24] showed that the contact normals distribution, $E(n)$, can be presented as follows:

$$
E(n)=\left(\frac{1}{2 \pi}\right)\left(1+\alpha \cos 2\left(\theta-\theta_{f}\right)\right)
$$

where $\alpha$ is the magnitude of anisotropy and $\theta_{f}$ is the major principal direction of the fabric tensor. The variations of the parameters $\alpha$ and $\theta_{f}$ represent the evolution of anisotropy in the granular mass. Experimental data shows that the shear strength of the granular material is a function of the magnitude of $\alpha$ and $\theta_{f}[1,17,25]$. The following equation is used to consider the effect of the induced anisotropy:

$$
\left(\frac{\sigma_{1}}{\sigma_{2}}\right)_{f} \propto\left(1+\left(\frac{1}{2}\right) \alpha \cos 2\left(\theta_{\sigma}-\theta_{f}\right)\right) .
$$

As previously mentioned, the shear strength in the granular medium is a function of inherent and induced anisotropy. The equation can predict the difference between samples due to the fabric which is a combination of the inherent and induced anisotropy as follows [26]:

$$
\left(\frac{\sigma_{1}}{\sigma_{2}}\right)_{f} \propto\left[\left(1+\left(\frac{1}{2}\right) \alpha \cos 2\left(\theta-\theta_{f}\right)\right) \cos 2\left(\beta_{i}-\beta_{\circ}\right)\right] .
$$


Another parameter that must be added to the above relation is the rolling strength of the granular material. Oda et al. [25] and Bardet [27] showed the importance of the rolling strength of the particles, especially in a $2 \mathrm{D}$ case. This effect is incorporated in the following form [26]:

$$
\begin{aligned}
\left(\frac{\sigma_{1}}{\sigma_{2}}\right)_{f} \propto[ & \left(1+\left(\frac{1}{2}\right) \alpha \cos 2\left(\theta-\theta_{f}\right)\right) \\
& \left.\times \cos 2\left(\beta_{i}-\beta_{\circ}\right) m \exp \left(\cos 2\left(\beta_{i}-\beta_{\circ}\right)\right)\right],
\end{aligned}
$$

where $m$ is a constant that depends on the interparticle friction angle $\phi_{\mu}$ and the shape of the particles. When the samples with equal densities are subjected to the shear loads, the difference in the shear strength due to the fabric can be attributed to (8).

\section{Verification of (8) with the Experimental Data}

In order to show the ability of (8) to represent the effect of the fabric on the shear strength, the predictions are compared with the experimental tests presented by Konishi et al. [25]. They conducted an experimental study on biaxial deformation of two-dimensional assemblies of rod-shaped photoelastic particle with oval cross section. The samples were confined laterally by a constant force of $0.45 \mathrm{kgf}$ and then compressed vertically by incremental displacement. Two types of particle shapes were used; one was $r_{1} / r_{2}=1.1$ and the other was $r_{1} / r_{2}=1.4$, in which $r_{1}$ and $r_{2}$ are the major and minor axes of cross section respectively. To consider the influence of friction, two sets of experiments were performed on these two particle shapes, one with nonlubricated particles of average friction angle of $52^{\circ}$ and the other with particles which had been lubricated with an average friction angle of $26^{\circ}$. The magnitude of the degree of anisotropy $\alpha$ and the major direction of the fabric $\theta_{f}$ are calculated by the following equations:

$$
\begin{aligned}
& A=\int_{0}^{2 \pi} E(\theta) \sin 2 \theta d \theta, \\
& B=\int_{0}^{2 \pi} E(\theta) \cos 2 \theta d \theta, \\
& \theta_{f}=\left(\frac{1}{2}\right) \arctan \left(\frac{A}{B}\right) .
\end{aligned}
$$

To show the ability of (8), the proportion of fabric with the shear strength variations is shown in Figure 1. The differences in the shear strength ratio at failure for different bedding angles are attributed to the differences in the developed anisotropic parameters. In other words, the combination of anisotropic parameters (for inherent and induced anisotropy) is proportional to the shear strength. The variation of righthand side of (8) is proportional to the variation of shear strength ratio for different bedding angles. The right-hand side of (8) is shown by fabric anisotropy in Figure 1. The effect of bedding angle on stress ratio at failure for the different interparticle friction angle $\phi_{\mu}$ is also shown in Figure 1.

\section{Incorporation of the Fabric and Its Evolution in the Yield Surface}

Muir Wood et al. [14] proposed the kinematic version of the Mohr-Coulomb yield surface as follows:

$$
f=q-\eta_{y}^{f} p_{o}
$$

where $q$ is the deviatoric stress and $\eta_{y}^{f}$ is the size of the yield surface. Muir Wood et al. [14] and Muir Wood [16] assumed that the soil is a distortional hardening material; hence, the current yield surface $\eta_{y}^{f}$ is a function of the plastic distortional strain $\varepsilon_{q}^{p}$, and, hence,

$$
\eta_{y}^{f}=\frac{\varepsilon_{q}^{p}}{c+\varepsilon_{q}^{p}} \eta^{p},
$$

where $\eta^{p}$ is a limit value of stress ratio which is equal to $M$ at the critical state, $\eta^{p}=M=q / p ; c$ is a soil constant.

Wood et al. [14] and Gajo and Muir Wood [15] developed the above equation to include the effect of state parameter $\psi=$ $e-e_{\mathrm{cr}}$, in which $e$ is the void ratio and $e_{\mathrm{cr}}$ is the magnitude of the void ratio on the critical-state line, as follows:

$$
\eta_{y}^{f}=\frac{\varepsilon_{q}^{p}}{c+\varepsilon_{q}^{p}}(M-k \psi),
$$

where $k$ is a constant.

Li and Dafalias [20] modified the effect of state parameter $\psi$ to account for a wide range of stress and void ratio as follows:

$$
\eta^{p}=M \exp \left(-n_{b} \psi\right)
$$

where $n_{b}$ is a material constant. Equation (7) can be modified as follows:

$$
\eta_{y}^{f}=\frac{\varepsilon_{q}^{p}}{c+\varepsilon_{q}^{p}} M \exp \left(-n_{b} \psi\right) .
$$

In the previous section, the shear strength was shown to be a function of inherent and induced anisotropy (see (8)). Thus, the effect of inherent and induced fabric anisotropy for triaxial case can be expressed as follows:

$$
\begin{aligned}
\eta_{y}^{f}= & \frac{\varepsilon_{q}^{p}}{c+\varepsilon_{q}^{p}}\left(1+\left(\frac{1}{2}\right) \alpha \cos 2\left(\theta_{f}-\theta_{\sigma}\right)\right) \\
& \times \cos 2\left(\beta_{i}-\beta_{\circ}\right) M \exp \left(-n_{b} \psi\right) .
\end{aligned}
$$

The magnitudes of $\alpha$ and $\theta_{f}$ approach a constant value in large shear strain $[26,28,29]$. The parameter $\cos 2\left(\beta_{i}-\beta_{\circ}\right)$ is easily obtained by back calculation but as a rough estimation, its value is close to the magnitude of the bedding angle $\cos \delta$ (for bedding angle $\delta$ between $15^{\circ}$ and $45^{\circ}$ ).

Equation (10) can be shown in the following form for multiaxial direction (or in the general form):

$$
f=\tau-\eta_{y}^{f} g(\theta) p_{o} .
$$

It is similar to the equation proposed by Pietruszczak and Mroz [6] and Lade [3] but in this formulation, another function is used for fabric and its evolution. 


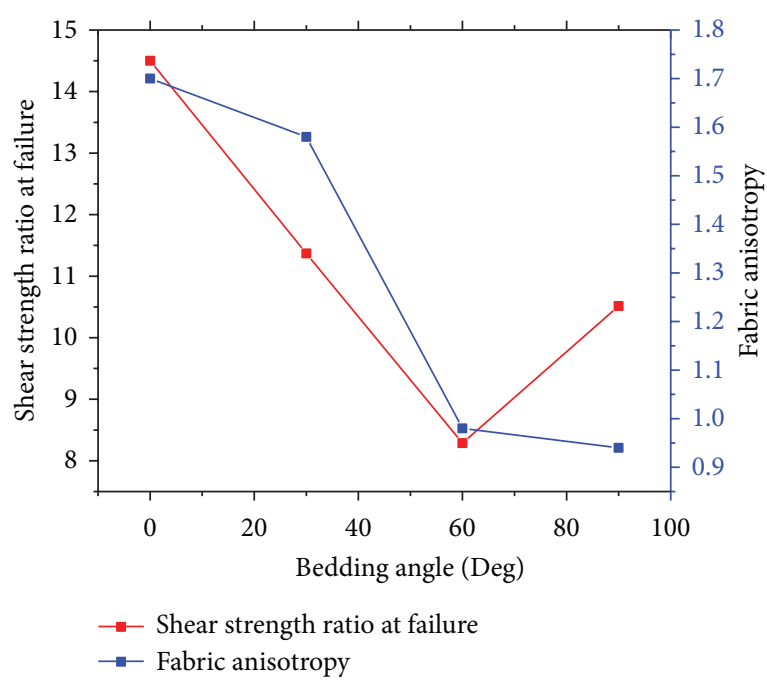

(a)

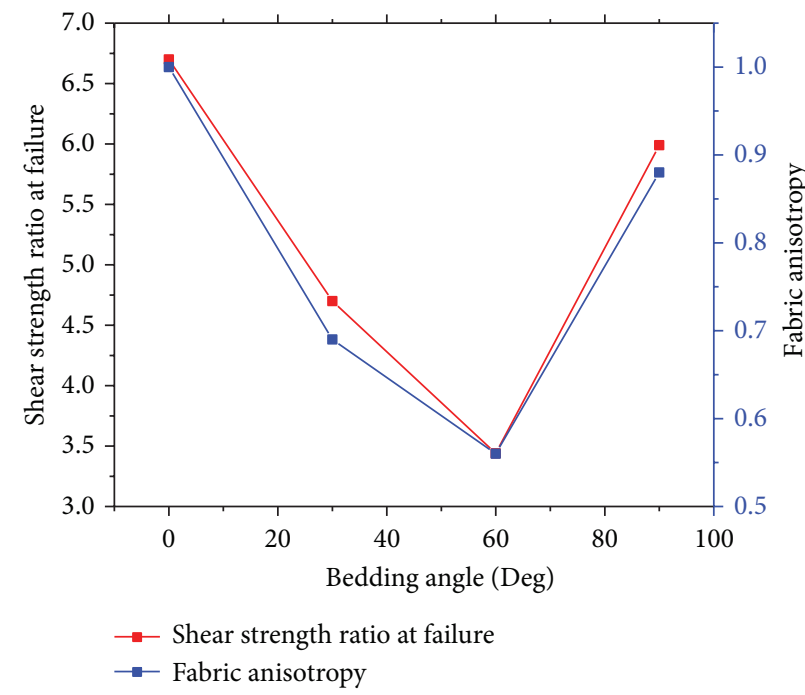

(c)

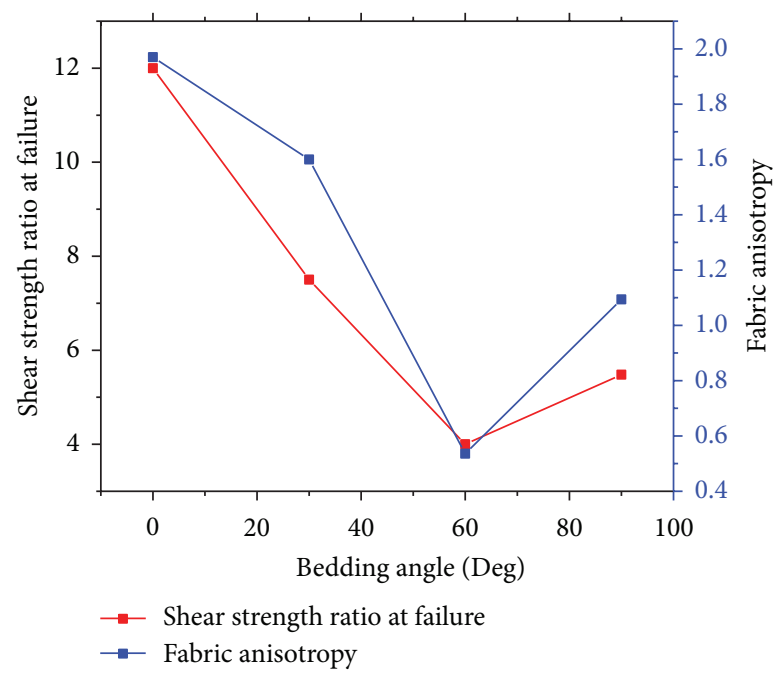

(b)

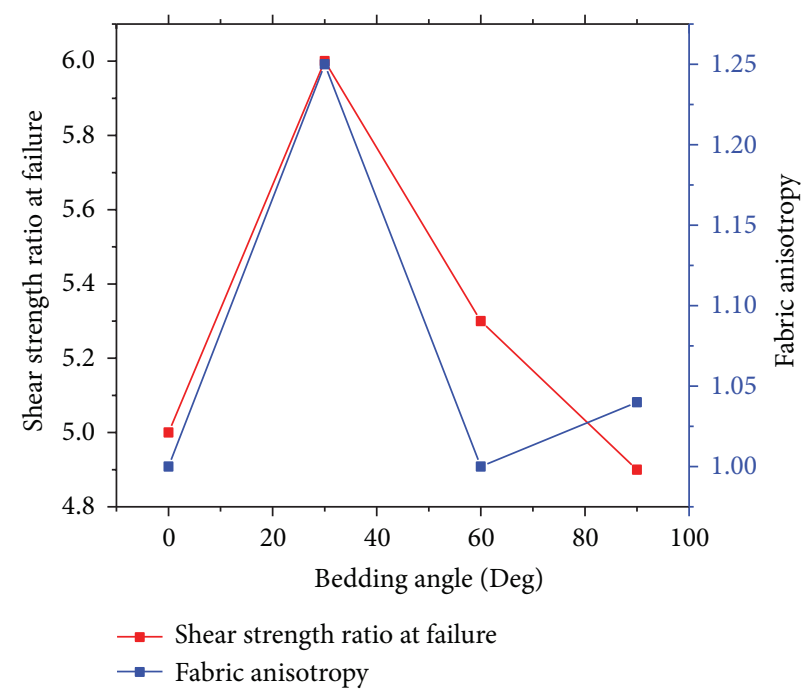

(d)

Figure 1: Samples with $\phi_{\mu}=52^{\circ}$ and $r_{1} / r_{2}=1.1(\mathrm{a}), \phi_{\mu}=26^{\circ}$ and $r_{1} / r_{2}=1.1(\mathrm{~b}), \phi_{\mu}=52^{\circ}$ and $r_{1} / r_{2}=1.4(\mathrm{c})$, and $\phi_{\mu}=26^{\circ}$ and $r_{1} / r_{2}=1.4$ (d).

\section{Fabric Evolution}

The parameters $\alpha$ and $\theta_{f}$ show the status of the fabric and its evolution. These parameters have a great influence on the behavior of the dilatancy equation. Shaverdi et al. [29] proposed an equation which can predict the magnitude of $\alpha$ and $\theta_{f}$ in the presence of the noncoaxiality between stress and fabric. This equation is obtained from the microlevel analysis. To calculate the $\alpha$ parameter, the magnitude of the shear to normal stress ratio on the spatially mobilized plane (SMP) must be determined. In the triaxial case, for example, $\tau$ / $p$ may be obtained from the following equation [30]:

$$
\frac{\tau}{p}=\sqrt{\frac{\sigma_{1}}{\sigma_{3}}}-\sqrt{\frac{\sigma_{3}}{\sigma_{1}}} .
$$

The parameters $\alpha$ and $\theta_{f}$ may be obtained from the following equations in the presence of noncoaxiality [29]:

$$
\begin{gathered}
\alpha=\frac{(\tau / p) \cos \phi_{\mu \mathrm{mob}}-\sin \phi_{\mu \mathrm{mob}}}{\sin \left(2 \theta_{f}+\phi_{\mu \mathrm{mob}}\right)-\left((\tau / p) \cos \left(2 \theta_{f}+\phi_{\mu \mathrm{mob}}\right)\right)}, \\
\dot{\theta}_{f}=\dot{\theta}_{\sigma}+\left(\frac{1}{2}\right) \cdot d \eta \cdot\left(\theta_{\sigma}-\theta_{f}\right)
\end{gathered}
$$

where the dot over $\theta$ shows the variation. The most important parameter in the above equation is the interparticle mobilized 


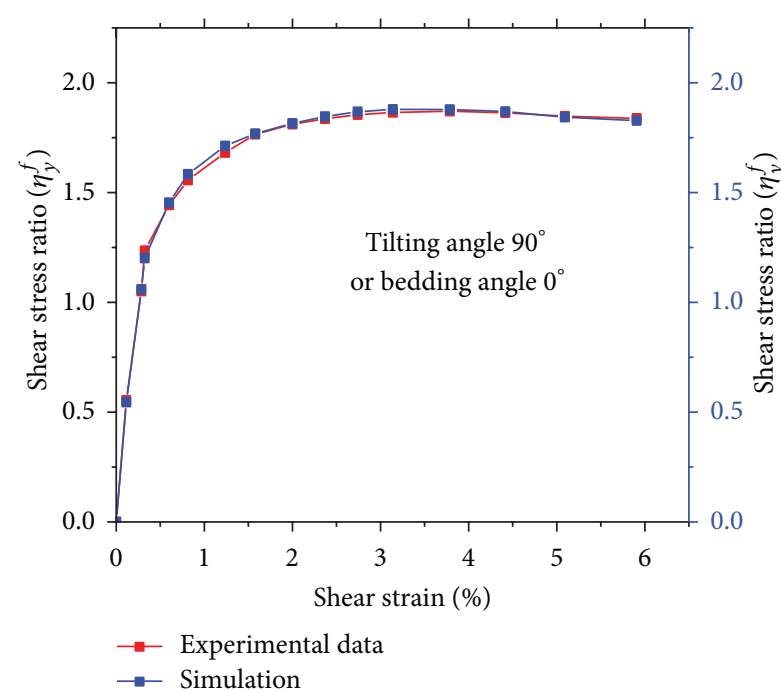

(a)

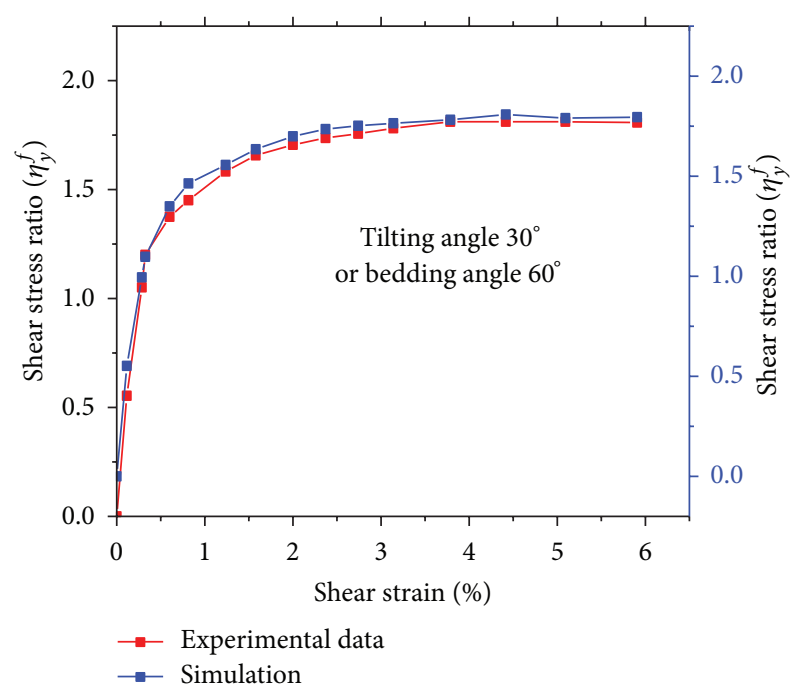

(c)

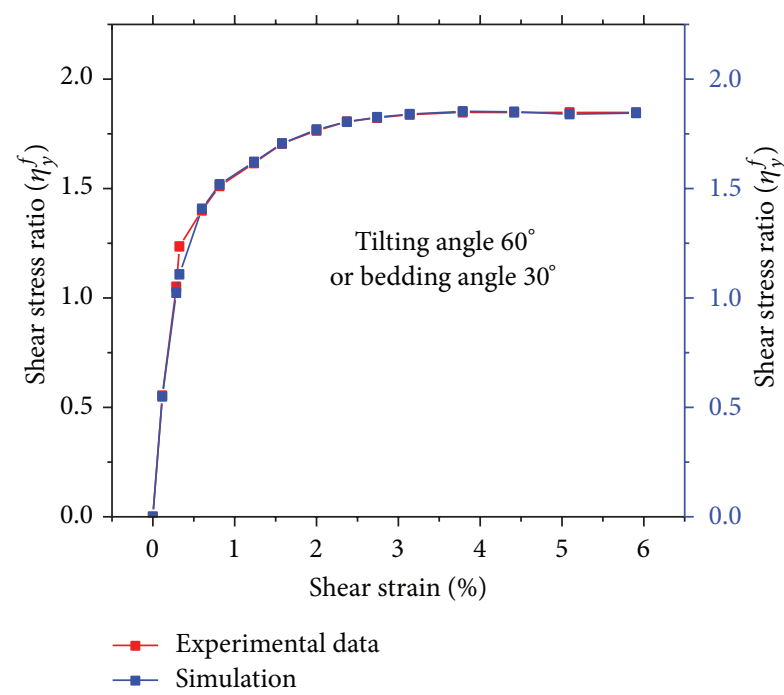

(b)

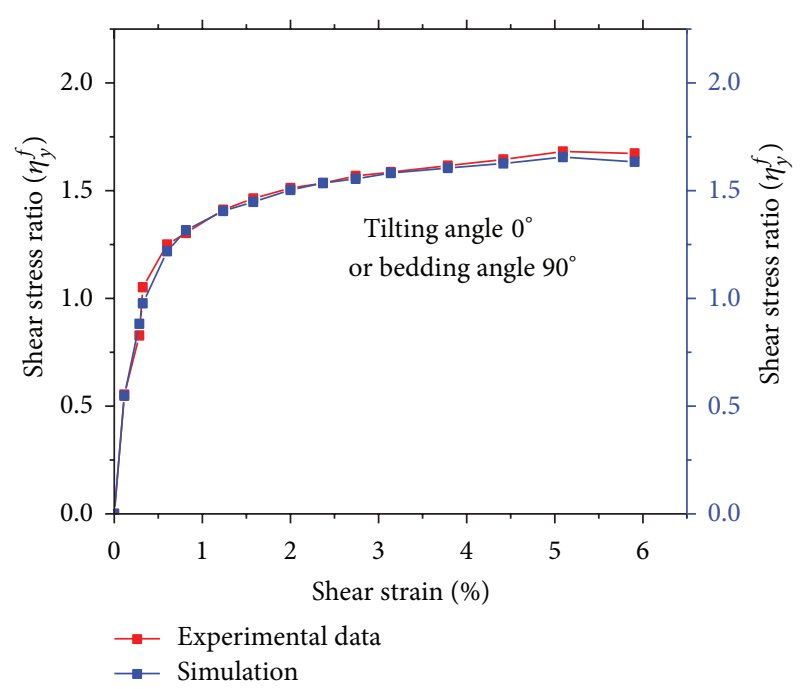

(d)

FIGURE 2: Comparison between experimental data and simulation by using (16) for the confining pressure $0.5 \mathrm{~kg} / \mathrm{cm}^{2}$.

friction angle, $\phi_{\mu \mathrm{mob}}$. This parameter is obtained from the following equation:

$$
\tan ^{-1}\left(\frac{\tau}{p}\right)=\frac{\theta_{\sigma}-\theta_{f}}{z}+\lambda\left(\frac{\dot{\varepsilon}_{v}}{\dot{\varepsilon}_{q}}\right)+\phi_{\mu \mathrm{mob}}
$$

where $z$ and $\lambda$ are material constants. Kuhn [28] and Shaverdi et al. [29] showed that the variation of $\alpha$ with the shear strain is similar to the variation of shear to normal stress ratio with shear strain.

\section{Verification of (16) with Experimental Data}

Oda et al. [17] conducted some experimental tests on Toyoura sand with an initial void ratio $0.67-0.68$ and the confining pressures $0.5 \mathrm{~kg} / \mathrm{cm}^{2}$ and $2 \mathrm{~kg} / \mathrm{cm}^{2}$ to study the effect of bedding angle with tilting angles $\delta=0^{\circ}, 30^{\circ}, 60^{\circ}$, and $90^{\circ}$. For better modeling, the constant $c$ for the plastic shear strain less than $1 \%$ must be modified as follows:

$$
c=0.001+0.001\left\langle 1-m^{\prime} \varepsilon_{q}^{p}\right\rangle,
$$

where $m^{\prime}$ is a constant which depends on confining pressure and $\langle\cdots\rangle$ stands for the positive values only. In this simulation, $m^{\prime}=1$ is taken into account for the confining pressure $0.5 \mathrm{~kg} / \mathrm{cm}^{2}$, and $m^{\prime}=6.5$ for the confining pressure $2.0 \mathrm{~kg} / \mathrm{cm}^{2}$.

Equation (17) can model the different behavior of the granular soils with the same confining pressure and initial void ratio (density) in which the only difference is due to the fabric and its evolution, as shown in Figures 2 and 3. 


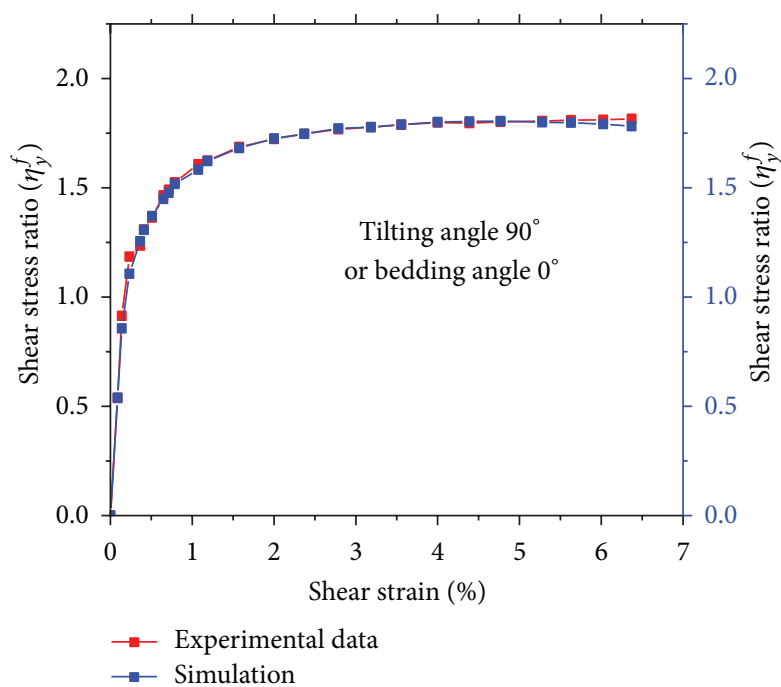

(a)

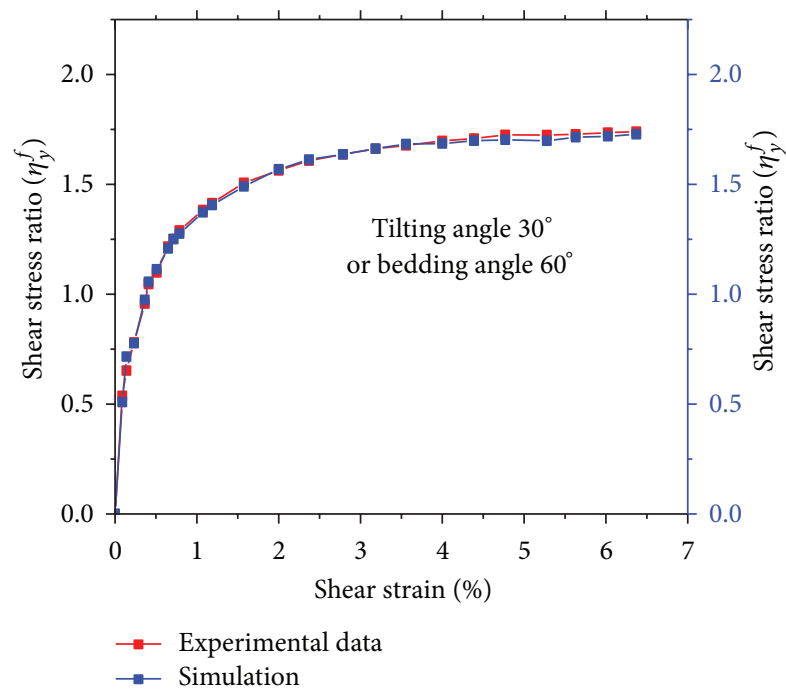

(c)

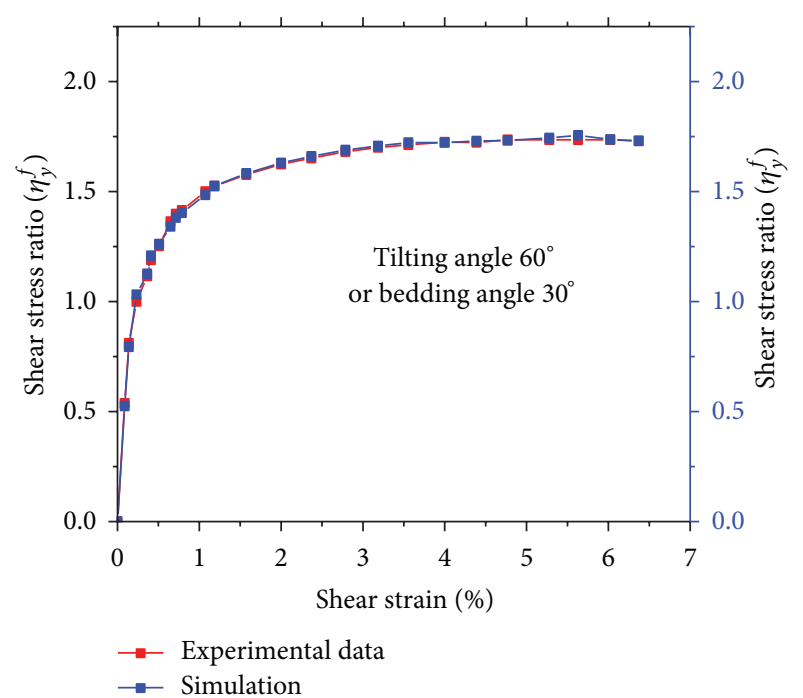

(b)

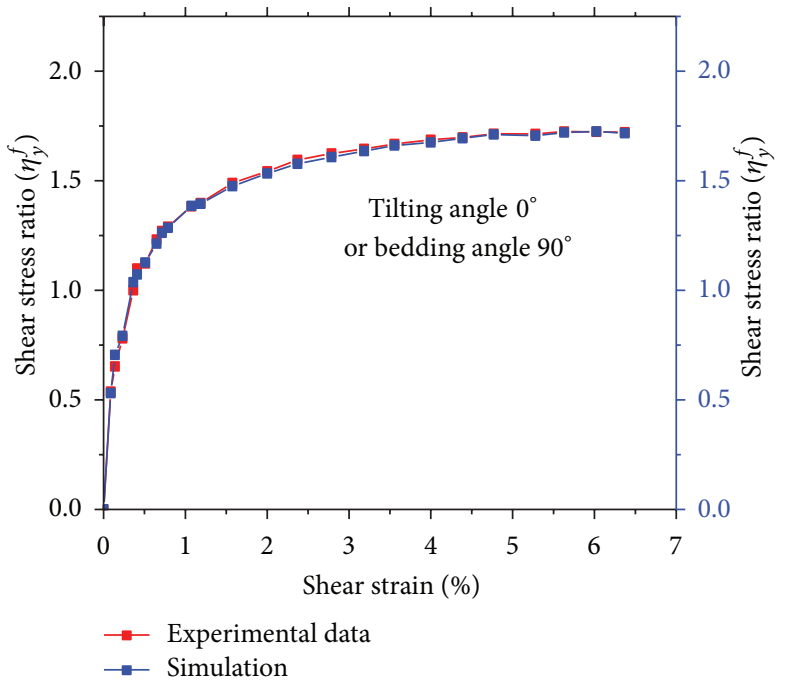

(d)

Figure 3: Comparison between experimental data and simulation by using (16) for the confining pressure $2.0 \mathrm{~kg} / \mathrm{cm}^{2}$.

\section{Conclusion}

An equation was proposed to include the effect of inherent and induced anisotropy. This relation was obtained by combining the effect of inherent and induced anisotropy. Rolling resistance is also included in this equation. The differences between the samples due to inherent and induced anisotropy were well captured by applying (8). Verifying the experimental data shows that this equation can predict the ratio of the shear strength at failure of granular materials in the presence of inherent anisotropy as good as possible. The effect of inherent anisotropy was incorporated by a single term $\cos 2\left(\beta_{i}-\beta_{0}\right)$. Induced anisotropy was also included by a simple term $\left(1+(1 / 2) \alpha \cos 2\left(\theta_{f}-\theta_{\sigma}\right)\right)$ in which $\alpha$ and $\theta_{f}$ can be easily calculated and obtained. The extended MohrCoulomb was developed to incorporate the effect of fabric and its evolution. Verification with the experimental tests demonstrated the validity of this formulation.

\section{References}

[1] M. Oda, "Initial fabrics and their relations to mechanical properties of granular materials," Soils and Foundations, vol. 12, no. 1, pp. 17-36, 1972.

[2] M. Oda, S. Nemat-Nasser, and J. Konishi, "Stress-induced anisotropy in granular mass," Soils and Foundations, vol. 25, no. 3, pp. 85-97, 1985.

[3] P. V. Lade, "Failure criterion for cross-anisotropic soils," Journal of Geotechnical and Geoenvironmental Engineering, vol. 134, no. 1 , pp. 117-124, 2008.

[4] R. Baker and C. S. Desai, "Induced anisotropy during plastic straining," International Journal for Numerical and Analytical Methods in Geomechanics, vol. 8, no. 2, pp. 167-185, 1984. 
[5] M. Pastor, "Modelling of anisotropic sand behaviour," Computers and Geotechnics, vol. 11, no. 3, pp. 173-208, 1991.

[6] S. Pietruszczak and Z. Mroz, "Formulation of anisotropic failure criteria incorporating a microstructure tensor," Computers and Geotechnics, vol. 26, no. 2, pp. 105-112, 2000.

[7] M. Oda, "Fabric tensor and its geometrical meaning," in Mechanics of Granular Materials: An Introduction, M. Oda and K. Iwashita, Eds., pp. 19-33, Balkema, Rotterdam, The Netherlands, 1999.

[8] M. Mehrabadi, S. Nemat-Nasser, and M. Oda, "On statistical description of stress and fabric in granular materials," International Journal For Numerical and Analytical Methods in Geomechanics, vol. 6, no. 1, pp. 95-108, 1982.

[9] Z. Gao, J. Zhao, and Y. Yao, "A generalized anisotropic failure criterion for geomaterials," International Journal of Solids and Structures, vol. 47, no. 22-23, pp. 3166-3185, 2010.

[10] Z. W. Gao and J. D. Zhao, "Efficient approach to characterize strength anisotropy in soils," Journal of Engineering Mechanics, vol. 138, no. 12, pp. 1447-1456, 2012.

[11] M. Oda and H. Nakayama, "Yield function for soil with anisotropic fabric," Journal of Engineering Mechanics, vol. 115, no. 1, pp. 89-104, 1989.

[12] P. Fu and Y. F. Dafalias, "Fabric evolution within shear bands of granular materials and its relation to critical state theory," International Journal for Numerical and Analytical Methods in Geomechanics, vol. 35, no. 18, pp. 1918-1948, 2011.

[13] P. Fu and Y. F. Dafalias, "Study of anisotropic shear strength of granular materials using DEM simulation," International Journal for Numerical and Analytical Methods in Geomechanics, vol. 35, no. 10, pp. 1098-1126, 2011.

[14] D. M. Wood, K. Belkheir, and D. F. Liu, "Strain softening and state parameter for sand modelling," Geotechnique, vol. 44, no. 2, pp. 335-339, 1994.

[15] A. Gajo and D. Muir Wood, "Severn-Trent sand: a kinematichardening constitutive model: the $q-p$ formulation," Geotechnique, vol. 49, no. 5, pp. 595-614, 1999.

[16] D. Muir Wood, Geotechnical Modelling, Taylor and Francis, 2004.

[17] M. Oda, I. Koishikawa, and T. Higuchi, "Experimental study of anisotropic shear strength of sand by plane strain test," Soils and Foundations, vol. 18, no. 1, pp. 25-38, 1978.

[18] M. Yoshimine, K. Ishihara, and W. Vargas, "Effects of principal stress direction and intermediate principal stress on undrained shear behavior of sand," Soils and Foundations, vol. 38, no. 3, pp. 179-188, 1998.

[19] R. G. Wan and P. J. Guo, "Drained cyclic behavior of sand with fabric dependence," Journal of Engineering Mechanics, vol. 127, no. 11, pp. 1106-1116, 2001.

[20] X. S. Li and Y. F. Dafalias, "Constitutive modeling of inherently anisotropic sand behavior," Journal of Geotechnical and Geoenvironmental Engineering, vol. 128, no. 10, pp. 868-880, 2002.

[21] X. S. Li and Y. F. Dafalias, "A constitutive, framework for anisotropic sand including non-proportional loading," Geotechnique, vol. 54, no. 1, pp. 41-55, 2004.

[22] Y. F. Dafalias and M. T. Manzari, "Simple plasticity sand model accounting for fabric change effects," Journal of Engineering Mechanics, vol. 130, no. 6, pp. 622-634, 2004.

[23] X. S. Li and Y. F. Dafalias, "Anisotropic critical state theory: role of fabric," Journal of Engineering Mechanics, vol. 138, no. 3, pp. 263-275, 2012.

[24] L. Rothenburg and R. J. Bathurst, "Analytical study of induced anisotropy in idealized granular materials," Geotechnique, vol. 39, no. 4, pp. 601-614, 1989.
[25] J. Konishi, M. Oda, and S. Nemat-Nasser, "Inherent anisotropy and shear strength of assembly of oval cross-section rods," in Proceedings of the IUTAM Symposium on deformation and failure of granular materials, Delft, The Netherland, 1982.

[26] H. Shaverdi, M. R. Taha, and F. Kalantary, "Effect of fabric on the shear strength of granular materials in biaxial compression," in Proceedings of the 7th International Conference on Micromechanics of Granular Media, pp. 615-618, Sydney, Australia, July 2013.

[27] J. P. Bardet, "Observations on the effects of particle rotations on the failure of idealized granular materials," Mechanics of Materials, vol. 18, no. 2, pp. 159-182, 1994.

[28] M. R. Kuhn, "Micro-mechanics of fabric and failure in granular materials," Mechanics of Materials, vol. 42, no. 9, pp. 827-840, 2010.

[29] H. Shaverdi, M. R. Taha, and F. Kalantary, "Evolution of fabric in the shearing process based on micromechanics," in Constitutive Modeling of Geomaterials, Q. Yang, J. Zhang, H. Zheng, and Y. Yao, Eds., pp. 469-475, Springer, Berlin, Germany, 2013.

[30] H. Matsuoka and H. Geka, "A stress-strain model for granular materials considering mechanism of fabric change," Soils and Foundations, vol. 23, no. 2, pp. 83-97, 1983. 


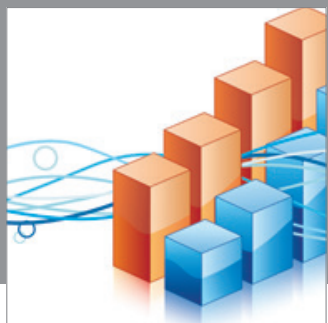

Advances in

Operations Research

mansans

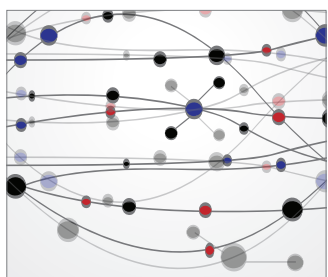

The Scientific World Journal
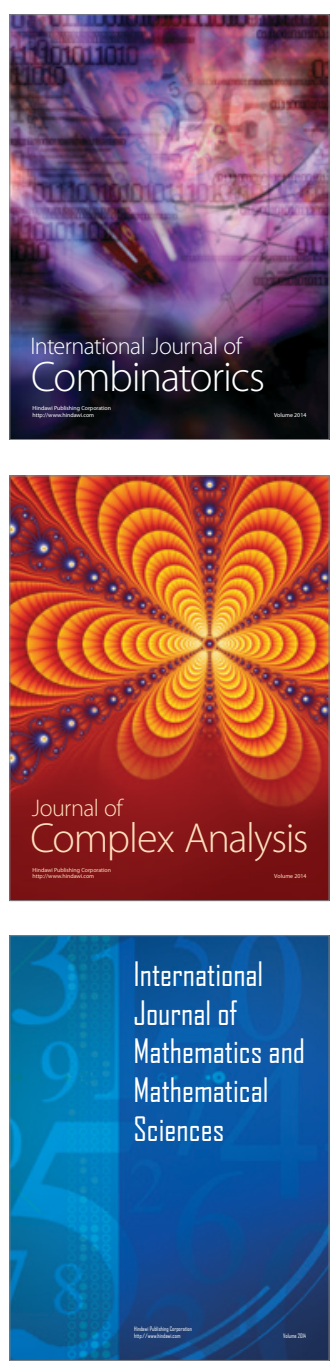
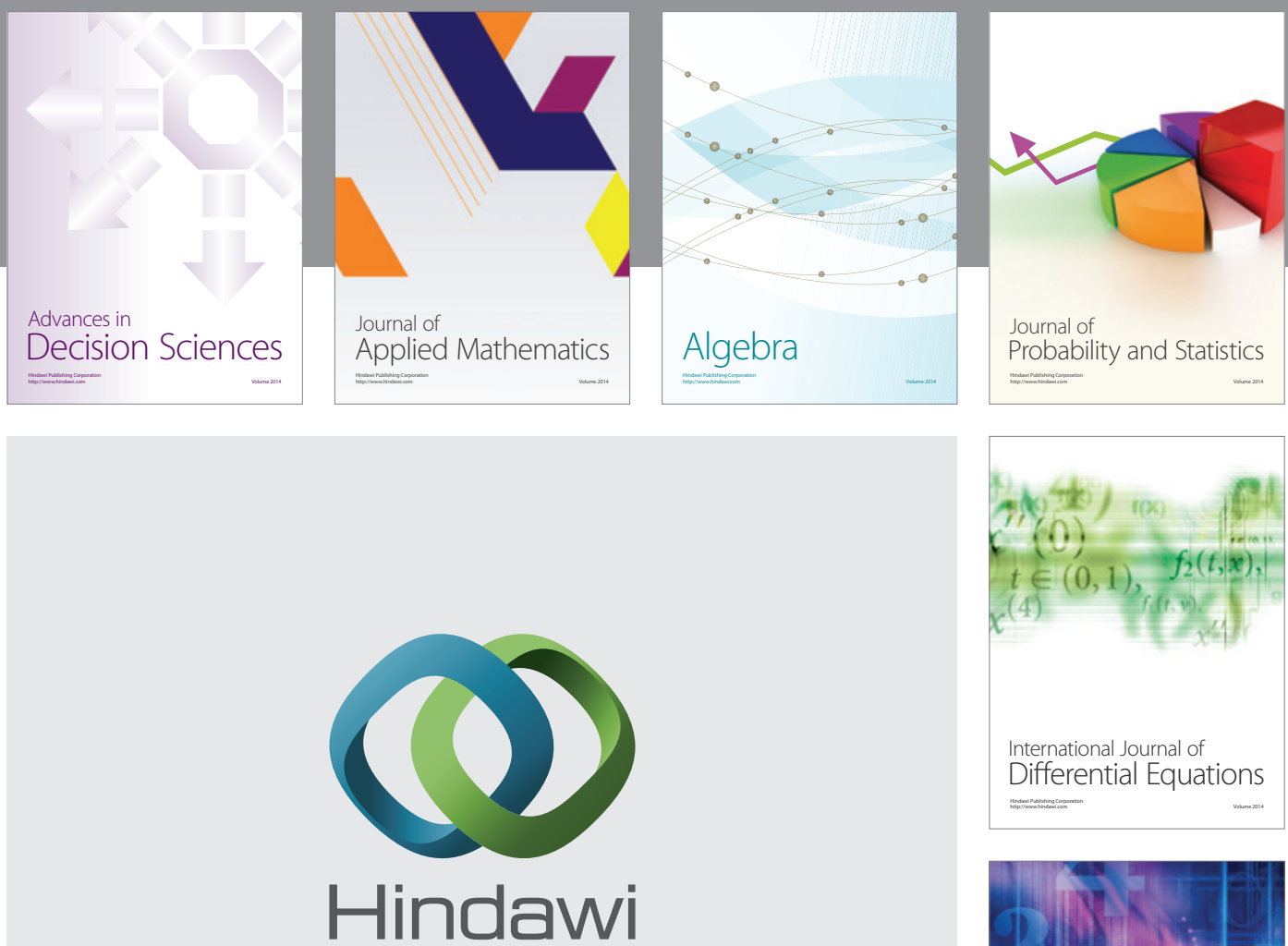

Submit your manuscripts at http://www.hindawi.com
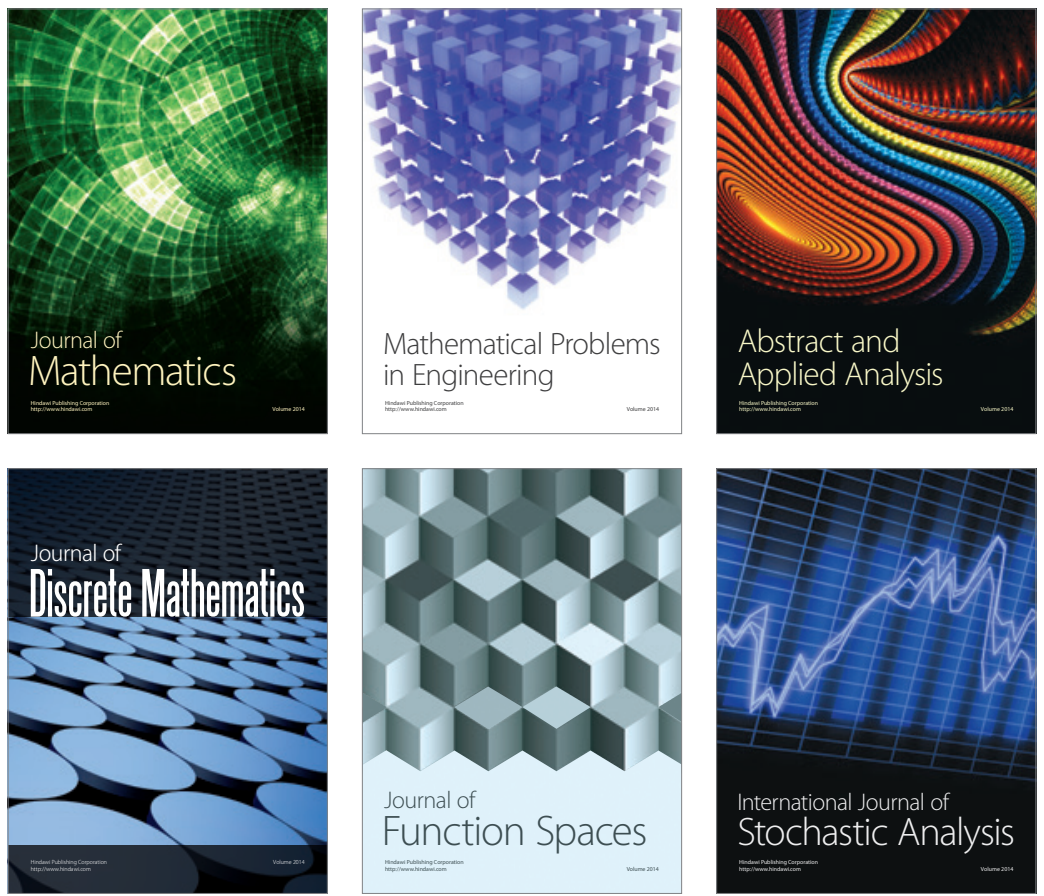

Journal of

Function Spaces

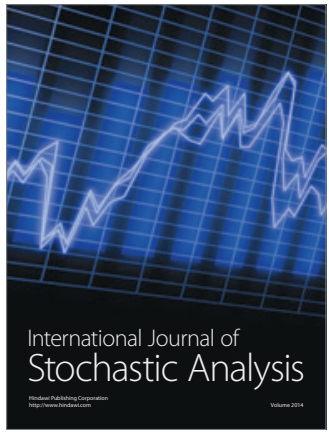

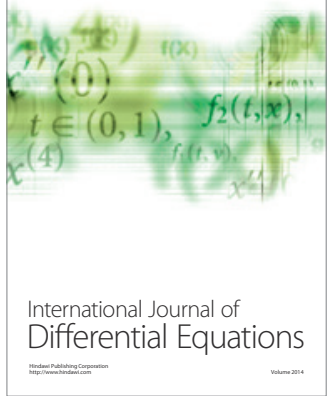
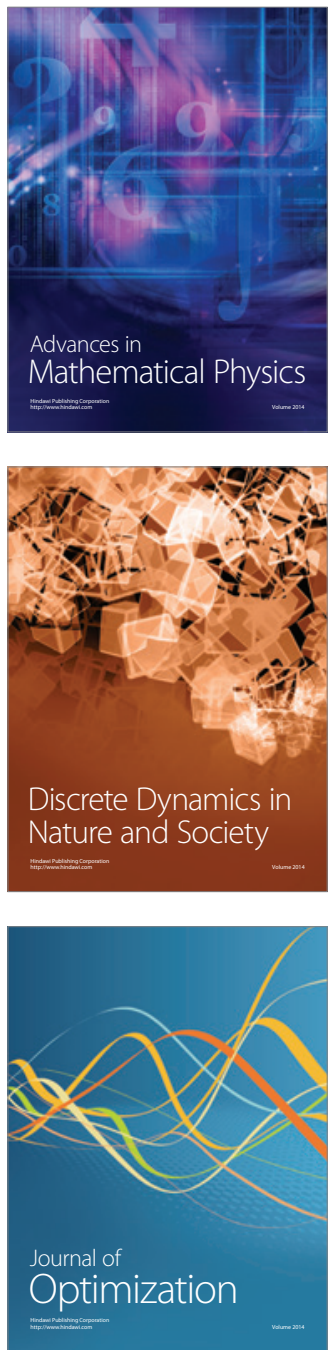